\title{
Integration and transmission of distributed deterministic neural activity in feed-forward networks
}

\author{
Yoshiyuki Asai ${ }^{\mathrm{a}, \mathrm{b}}$, Alessandro E. P. Villa ${ }^{\mathrm{b}, *}$ \\ ${ }^{a}$ Open Biology Unit, Okinawa Institute of Science and Technology. Okinawa, Japan \\ ${ }^{b}$ Neuroheuristic Research Group, HEC-ISI, University of Lausanne, Switzerland \\ ${ }^{c}$ INSERM UMRS 836; Université Joseph Fourier, Grenoble, France
}

\begin{abstract}
A ten layers feed-forward network characterized by diverging/converging patterns of projection between successive layers of regular spiking (RS) neurons is activated by an external spatiotemporal input pattern fed to Layer 1 in presence of stochastic background activities fed to all layers. We used three dynamical systems to derive the external input spike trains including the temporal information, and three types of neuron models for the network, i.e. either a network formed either by neurons modeled by exponential integrate-and-fire dynamics (RS-EIF, Fourcaud-Trocmé et al., 2003), or by simple spiking neurons (RS-IZH, Izhikevich, 2004) or by multiple-timescale adaptive threshold neurons (RS-MAT, Kobayashi et al., 2009), given five intensities for the background activity. The assessment of the temporal structure embedded in the output spike trains was carried out by detecting the preferred firing sequences for the reconstruction of de-noised spike trains (Asai and Villa, 2008). We confirmed that the RS-MAT model is likely to be more efficient in integrating and transmitting the temporal structure embedded in the external input. We observed that this structure could be propagated not only up to the 10-th layer but in some cases it was retained better beyond the 4-th downstream layers. This study suggests that diverging/converging network structures, by the propagation of synfire activity, could play a key role in the transmission of complex temporal patterns of discharges associated to deterministic nonlinear activity.
\end{abstract}

Keywords: preferred firing sequences, synfire chain, spatiotemporal firing patterns

\section{Introduction}

Spike trains are sequences of the timing of the occurrences of neuronal action potentials. Experimental evidence of deterministic chaotic properties in spike trains obtained from in vivo extracellular recordings (Celletti and Villa, 1996a,b; Villa et al., 1998b; Segundo et al., 1998; Celletti et al., 1999) suggest that a neuronal network can be considered as a complex nonlinear dynamical system able to exhibit chaotic dynamics (van Vreeswijk and Sompolinsky, 1996; Segundo, 2003). Each neuron of the network is also likely to receive background activities whose origin is unspecified or unknown and its activity is often represented by stochastic occurrences of spikes. Thus, it is possible to assume that in addition to stochastic background activity a network may receive inputs characterized by an embedded temporal structure, which is somehow associated to a deterministic nonlinear system. Diverging/converging feed-forward neuronal networks are able to transmit information with great temporal accuracy, emphasizing their characteristic to exhibit synchronous firing in one layer for they were termed synfire chains (Abeles, 1982a). The question whether and to what extent complex asynchronous temporal structure can be propa-

\footnotetext{
${ }^{*}$ Corresponding author

Email addresses: yoshiyuki.asai@oist.jp (Yoshiyuki Asai), avilla@neuroheuristic.org (Alessandro E. P. Villa)

URL: http: //www.neuroheuristic.org/ (Alessandro E. P. Villa)
}

gated in a reliable way by means of diverging/converging feedforward neuronal networks is important given the property of compositionality modeled by dynamic binding of these networks (Abeles et al., 2004; Kumar et al., 2008; Schrader et al., 2011). Previous studies (Tetko and Villa, 1997; Asai et al., 2006; Asai and Villa, 2008) showed that spikes related to deterministic nonlinear dynamics embedded in noisy time series could be detected by applying algorithms aimed at finding preferred firing sequences with millisecond order time precision. Moreover, the characteristics of the transfer function of the neuron model and the statistical feature of the the background activity may affect heavily the propagation of temporal information through the synapses (Asai et al., 2008).

In the current paper we extend our previous analysis (Asai and Villa, 2010). Each neuron in the input layer of the synfire chain receives only randomly selected fractions of the spike train associated to deterministic chaotic dynamics. We examined the ability of the neural network to transmit the temporal structure embedded in the external input spike trains in the presence of background activity with various intensities. The detection of preferred firing sequences by pattern grouping algorithm (Villa and Tetko, 1999; Tetko and Villa, 2001b; Abeles and Gat, 2001) in all layers of the network revealed a reliable propagation of temporal information through the layers. In some cases with stronger background activity we could observe the integration of fractions of the temporal information fed to the neurons of the first layer up to the fourth layer. In addition we 
present evidence that adaptive threshold neurons (Kobayashi et al., 2009) can maintain and integrate the distributed temporal structures in more robust way against the noise than the exponential integrate-and-fire (RS-EIF) neuron model (FourcaudTrocmé et al., 2003) and simple spiking neurons (Izhikevich, 2003, 2004).

\section{Methods}

\subsection{Spiking neuron model}

We adopted three neuron models to simulate the dynamics of regular spiking (RS) neurons. The first is the exponential integrate-and-fire (RS-EIF) model (Fourcaud-Trocmé et al., 2003), which is known to reproduce the dynamics of a simple conductance-based model extremely well. In this model the membrane potential $V$ dynamics is given by

$$
\begin{aligned}
C \frac{d V}{d t} & =-g_{L}\left(V-V_{L}\right)+\psi(V)+I(t), \\
\psi(V) & =g_{L} \Delta_{T} \exp \left(\frac{V-V_{T}}{\Delta_{T}}\right)
\end{aligned}
$$

where $C$ is the membrane capacitance, $g_{L}$ is the leak conductance, $V_{L}$ is the leak potential, $I(t)$ is the external synaptic current, and $\psi(V)$ is a function of the voltage that describes the spike generating currents. Notice that with $\psi(V)=0$ the model becomes a simple leaky integrate-and-fire. $\Delta_{T}$ is a parameter called the spike slope factor (in $\mathrm{mV}$ ) representing the voltage sensitivity of the spiking current and $V_{T}$ is the threshold voltage. If the input current exceeds the threshold a spike is fired instantaneously and the membrane potential is reset to a voltage $V_{r}$. The parameters were set to the values indicated by FourcaudTrocmé et al. (2003): $V_{L}=-65 \mathrm{mV}, V_{T}=-59.9 \mathrm{mV}, V_{r}=-68$ $\mathrm{mV}, C=1 \mu \mathrm{F} / \mathrm{cm}^{2}, g_{L}=0.1 \mathrm{mS} / \mathrm{cm}^{2}$, and $\Delta_{T}=3.48 \mathrm{mV}$. The passive membrane time constant is $\tau_{m}=C / g_{L}=10 \mathrm{~ms}$.

The second model is a simple spiking neuron (RS-IZH) model (Izhikevich, 2004) described by the following equations:

$$
\begin{aligned}
& \frac{d v}{d t}=0.04 v^{2}+5 v+140-u+I(t) \\
& \frac{d u}{d t}=a(b v-u)
\end{aligned}
$$

where $v$ represents the membrane potential $[\mathrm{mV}], u$ is a membrane recovery variable, $a$ and $b$ control the time scale of the membrane potential dynamics. $I(t)$ is a total synaptic current given to the neuron. When $v \geq+30 \mathrm{mV}$, a discontinuous resetting $v \leftarrow c$ and $u \leftarrow u+d$ follows as a hyperpolarization after a spike. The parameters were set as $a=0.02, b=0.2, c=-65$, $d=8$ (Izhikevich, 2004).

The third model is a multiple-timescale adaptive threshold (RS-MAT) neuron model (Kobayashi et al., 2009) whose membrane potential dynamics follows a non-resetting leaky integrator,

$$
\tau_{m} \frac{d V}{d t}=-V(t)+R I(t)
$$

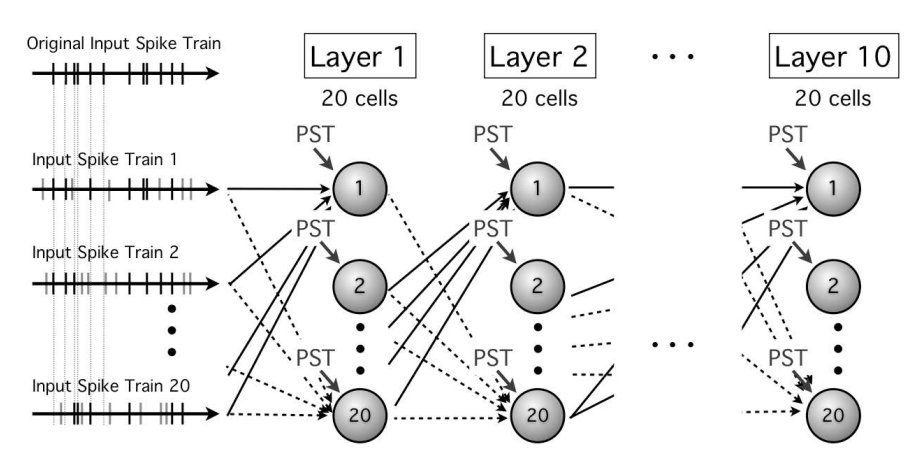

Figure 1: Schematic representation of the convergent/divergent feed-forward circuit formed by 10 neuronal layers. Each layer includes 20 cells. Each cell receives 15 afferents and a independent Poissonian spike train (PST).

where $\tau_{m}, V$ and $R$ are the membrane time constant, membrane potential and membrane resistance, respectively. $I(t)$ is the external synaptic current received by the neuron. A spike is generated if $V(t) \geq \theta(t)$,

$$
\begin{aligned}
\theta(t) & =\omega+H_{1}(t)+H_{2}(t), \\
\frac{d H_{1}}{d t} & =-H_{1} / \tau_{1}, \\
\frac{d H_{2}}{d t} & =-H_{2} / \tau_{2},
\end{aligned}
$$

where $\omega$ is the resting value. $H_{1}$ and $H_{2}$ are components of the fast and slow threshold dynamics (characterized by decaying time constants $\tau_{1}$ and $\tau_{2}$, respectively) which have a discrete jump when $V(t) \geq \theta(t)$,

$$
H_{1}=H_{1}+\alpha_{1}, \quad H_{2}=H_{2}+\alpha_{2} .
$$

The parameters were set to values $\tau_{m}=5 \mathrm{~ms}, R=50 \mathrm{M} \Omega$, $\omega=19 \mathrm{mV}, \tau_{1}=10 \mathrm{~ms}, \tau_{2}=200 \mathrm{~ms}, \alpha_{1}=37 \mathrm{mV}$, and $\alpha_{2}=2$ $\mathrm{mV}$.

\subsection{Neural network}

We consider a diverging/converging neural network composed of 10 layers (Fig. 1). Each layer includes 20 neurons. All neurons in a network are identical and are either RS-EIF, RS-IZH or RS-MAT models. Each neuron of Layer 1 receives afferents carrying temporal information. The external input is represented by 15 spike trains derived from selected dynamical systems described below. Each neuron belonging to Layer 2 and further downstream in the network receives afferents from 15 neurons randomly selected among those of the immediately upstream layer. All connections were hardwired, and no synaptic plasticity was taken into account. Explicit synaptic transmission delays were randomly and uniformly distributed in the range $0.7-1.3 \mathrm{~ms}$. In addition to these network inputs each neuron of any layer receives a background activity represented by an independent Poissonian spike train. In a single execution of a simulation, the mean firing rate of the background activity is the same for all neurons and is selected in the range [300, 350, 400, 450, 500] spikes/s. 

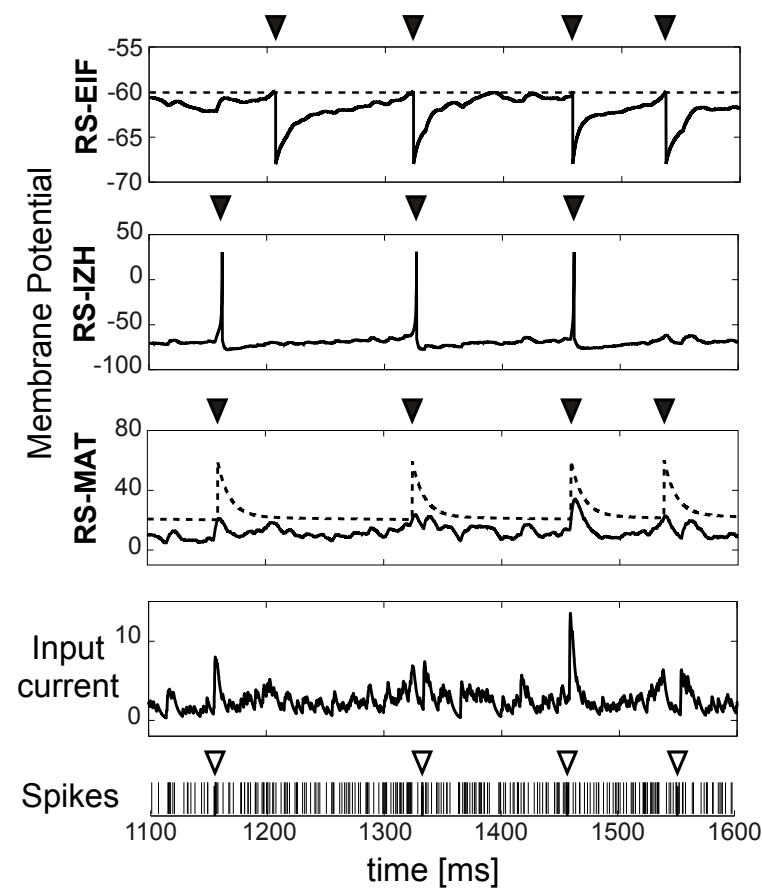

Figure 2: Dynamics of neuron models of cells of Layer 1. Membrane potential (solid line) of RS-EIF, RS-IZH and RS-MAT neurons as a function of time. For the RS-EIF and RS-MAT models the dashed line corresponds to the threshold. Filled triangles indicate occurrences of spikes. The input current corresponds to the total synaptic current produced by the incoming spikes shown at the bottom panel (not corrected by the scaling factor chracteristic for each model). In the bottom panel the spikes derived from the external input are tagged by open triangles. All other spikes belong to the Poissonian background activity (with mean firing rate 500 spikes/s in this example).

The total external synaptic current $I(t)$ produced by the synaptic afferences on a post-synaptic neuron was defined as follows:

$$
I(t)=-A \sum_{k} g_{s y n}\left(t-t_{k}\right),
$$

where $A$ is a scaling factor of the intensity of the synaptic transmission and $t_{k}$ represents time when the $k$-th spike arrives to the neuron. $g_{s y n}$ is the post synaptic conductance represented by

$$
g_{s y n}(t)=C_{0} \frac{e^{-t / \tilde{\tau}_{1}}-e^{-t / \tilde{\tau}_{2}}}{\tilde{\tau}_{1}-\tilde{\tau}_{2}},
$$

where $\tilde{\tau}_{1}$ and $\tilde{\tau}_{2}$ are rise and decay time constants given by 0.17 and $4 \mathrm{~ms}$, respectively. $C_{0}$ is a coefficient used to normalize the maximum amplitude of $g_{s y n}(t)$ to 1 . A single synaptic current given to a neuron is not strong enough to evoke post-synaptic neuronal discharges. Numerical integration was done by the fourth order Runge-Kutta numerical integration method with $0.01 \mathrm{~ms}$ time steps for all cases and the total simulation duration corresponded to 2,000 seconds. The scaling factor of the intensity of the synaptic transmission was adjusted in order to obtain a firing rate of 5 spikes/s in Layer 1 neurons stimulated with an original input spike train and background intensity of 400 spikes $/ \mathrm{s}$. The value of $A$ was equal to $0.0630,0.9000$ and 0.0954 and for RS-EIF, RS-IZH and RS-MAT, respectively.
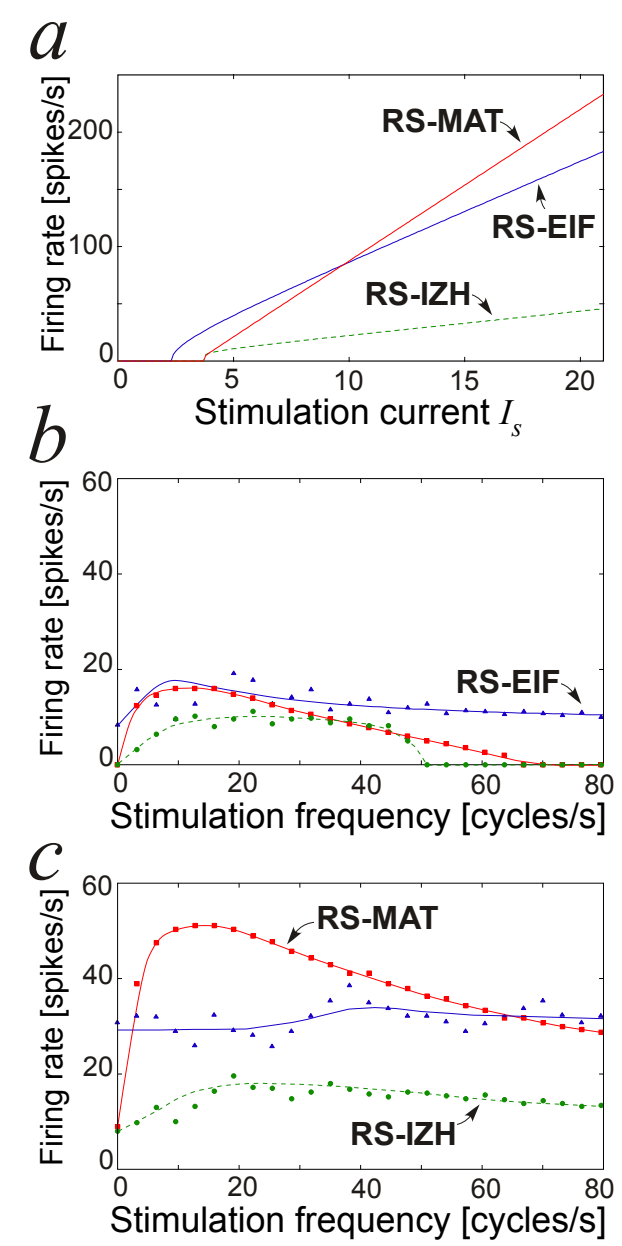

Figure 3: Panel $a$ : $I-F$ curves of the RS-EIF, RS-IZH and RS-MAT neurons for a constant input current. The stimulation current $I_{S}$ current is not scaled; the scaling factors adapted for each model are indicated in the text. Panel $b$ : Firing frequency as a function of the frequency of a sinusoidal input whose maximum amplitude is $I_{S}=4.6$. Panel $c$ : Firing frequency as a function of the frequency of a sinusoidal input whose maximum amplitude is $I_{S}=7.4$. Blue interpolated lines and filled triangles correspond to RS-EIF. Dashed interpolated lines and filled circles correspond to RS-IZH. Red interpolated lines and filled squares correspond to RS-MAT.

The dynamics of the neuron models as a function of their inputs is illustrated by Fig. 2. Features of the the three models are illustrated by the $I-F$ curves, i.e. the firing frequency of a neuron as a function of a constant input current (DC current) whose intensity $I_{S}$ is such that $I(t)=-A I_{S}$ in the absence of any synaptic input, where $A$ is the scaling factor. (Fig. 3a). In Figure $3 \mathrm{a}$ it appears that the RS-EIF neuron starts to fire with $I_{S}=2.3$ and RS-IZH and RS-MAT with $I_{S}=3.7$. These values have been selected to show the behavior of the models to a sinusoidal input with maximum amplitude equal to $I_{S}=4.6$ (Fig. 3b) and $I_{S}=7.4$ (Fig. 3c). Notice that RS-MAT and RSIZH have an almost flat response curve to the sinusoidal input, but RS-MAT shows a clear-cut response peak for a sinusoidal input near 12 cycles $/ \mathrm{s}$. 


\subsection{Dynamical systems for generation of the external input}

An external input carrying precise temporal information was generated from three nonlinear dynamical systems. We adopted Zaslavskii map, Ikeda map and Chen's system. These systems exhibit chaotic dynamics with certain parameter values which were selected to create the external input time series.

The Zaslavskii map is defined by

$$
\begin{aligned}
x_{n+1}= & x_{n}+v\left(1+\mu y_{n}\right)+\xi v \mu \cos x_{n} \\
& (\bmod .2 \pi), \\
y_{n+1}= & e^{-\gamma}\left(y_{n}+\xi \cos x_{n}\right),
\end{aligned}
$$

where $x, y \in \mathbf{R}$, and $\mu=\frac{1-e^{-\gamma}}{\gamma}, v=\frac{4}{3} \cdot 100, \gamma=3.0, \xi=0.1$. The initial conditions were set to $x_{0}=y_{0}=0.3$. The iterative calculation generated the time series $\left\{x_{n}\right\}$ used for the external input.

The Ikeda map is the quadratic mapping defined as follows,

$$
\begin{aligned}
& x_{n+1}=p+\mu\left(x_{n} \cos \theta-y_{n} \sin \theta\right), \\
& y_{n+1}=\mu\left(y_{n} \cos \theta+x_{n} \sin \theta\right)
\end{aligned}
$$

where $\theta=k-a /\left(1+x_{n}^{2}+y_{n}^{2}\right)$, and $a=6.0, k=0.4, p=1.0, \mu=$ 0.9 . Initial conditions were $x_{0}=y_{0}=0.3$. The sequence $\left\{x_{n}\right\}$ is used for the external input.

The Chen's system are formulated by three equations,

$$
\begin{aligned}
& \frac{d x}{d t}=a(y-x), \\
& \frac{d y}{d t}=(c-a) x-x z+c y, \\
& \frac{d z}{d t}=x y-b z,
\end{aligned}
$$

where $a=35.0, b=3.0, c=28.0$, and $x(0)=y(0)=3.0$ for initial conditions. We considered a Poincaré map where the Poincaré section was defined by $\frac{d x}{d t}=0$, and the sequence of $z(t)$ on the section was traced, referred to as $\left\{x_{n}\right\}$ hereinafter and used for the external input.

\subsection{Simulated input spike trains}

A new time series $\left\{w_{n}\right\}$ corresponding to the sequence of the inter-spike-intervals was derived from the external input time series $\left\{x_{n}\right\}$, following $w_{n}=x_{n+1}-x_{n}+K$, where $K=$ $-\min \left\{\left(x_{n+1}-x_{n}\right)\right\}+0.1$ is a constant to make sure $w_{n}>0$. The sequence $\left\{w_{n}\right\}$ was rescaled with a precision of $1 \mathrm{~ms}$ to an average rate of 5 events $/ s$, for the sake of comparison with neurophysiological firing rates of 5 spikes $/ \mathrm{s}$. We calculated $N=10,000$ points of the rescaled time series, now termed $\left\{w_{n}^{\prime}\right\}$, corresponding to a duration $L=2,000 \mathrm{~s}$. This time series $\left\{w_{n}^{\prime}\right\}$ is also termed the original external input and the points are assumed to correspond to spike occurrences.

The original external input as such was never used to activate a neuron of Layer 1 . We select at random (uniformly distributed) $\varepsilon \times N(0 \leq \varepsilon \leq 1)$ spikes from the original external input. The remaining spikes were deleted, thus yielding a sparse input spike train, where $\varepsilon$ is called "copy probability" and takes a value from 0 to 1 . The sparse input spike train was merged with a Poissonian spike train with mean firing rate
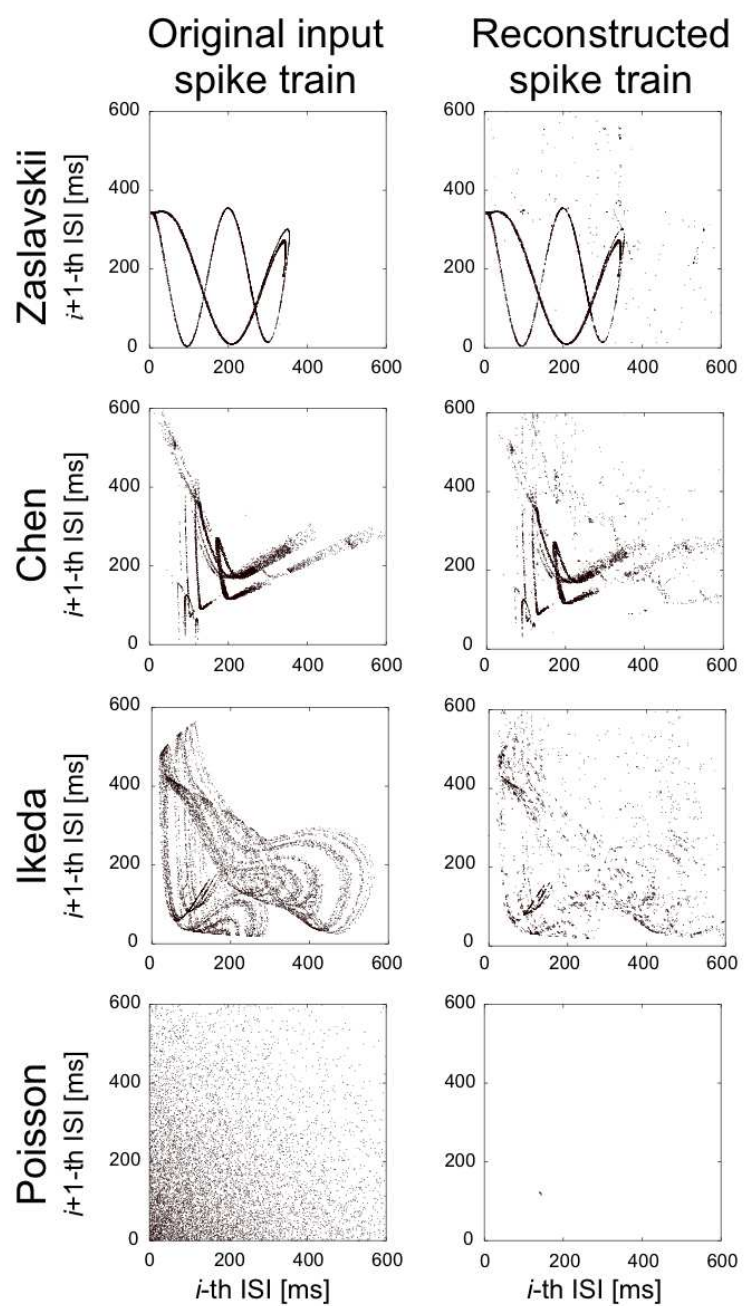

Figure 4: (Left column) Return maps of the original input spike trains derived from three dynamical systems with copy probability $\varepsilon=1$ (i.e., the original input spike train without any noise or deletion) are presented with the axes scaled in $m s$ time units. The bottom panel shows a return map of a pure Poissonian spike train (i.e. $\varepsilon=0$ ). (Right column) Return maps of reconstructed spike trains from the original input spike trains shown in the left column.

$N(1-\varepsilon) / L$ spikes $/ s$ in order to keep the average rate close to 5 spikes $/ \mathrm{s}$ and duration equal to $2,000 \mathrm{~s}$. This spike train whose sparseness is determined by the copy probability $\varepsilon$ correspond to a single "input spike train" fed to a Layer 1 neuron. This procedure is repeated 20 times for a given parameter $\varepsilon$, so to generate 20 different input spike trains. Notice that if $\varepsilon=1$ all input spike trains are identical to the original external input spike train, and if $\varepsilon=0$ all input spike trains are independent Poissonian spike trains. In this study we used $\varepsilon=1,0.5$ and 0.3. Return maps, a plot of the $(i+1)$-th inter-spike-interval against the $i$-th inter-spike-interval, of the original input spike trains were shown in the left panels of Fig. 4.

\subsection{Pattern detection and reconstruction of time series}

Subsets of spike trains were obtained by using the Pattern Grouping Algorithm (PGA) (Villa and Tetko, 1999; Tetko and Villa, 2001b,c,a; Abeles and Gat, 2001) as follows. Although 
PGA has a capability to be applied to a multivariate spike train, we applied PGA to a single cell activity, comparing the patterns detected in its input spike train and those detected in its output spike train. Firing sequences repeating at least 5 times and above the chance level $(p=0.05)$ are detected by PGA. The maximum interval between the first and the last spike of the pattern was set to $\leq 600 \mathrm{~ms}$. Given a maximum allowed jitter in spike timing accuracy $( \pm 3 \mathrm{~ms})$ clusters of firing sequences are represented by a template pattern. For example, if 9 triplets (i.e., spike sequences formed by 3 spikes) belonging to the same cluster were detected by PGA, a subset of the original spike train that includes 27 spikes $(=9 \times 3)$ can be determined. Rearranging all these spikes according to their time of firing, they formed a new spike train which is referred to as "reconstructed spike train" (Asai et al., 2006; Asai and Villa, 2008). Figure 4 shows the return map of reconstructed spike trains of the original input spike trains. With a copy probability $\varepsilon=1$, which means in the absence of any noise or deletion of any point of the external input time series, we found that $92 \%$ of the original external input spike train derived from Zaslavskii map are included in the reconstructed spike train. In the cases of Chen and Ikeda maps the reconstructed spike trains included $86 \%$ and $58 \%$ events, respectively, of the original external input spike train. In the case $\varepsilon=0$ no embedded temporally organized activity is present in the external input spike train and the reconstructed time series should contain zero points. We observed that with input represented only by pure Poissonian firing at an average rate of 5 spikes $/ s$ the reconstructed spike train included only $0.4 \%$ spikes of the original series (Fig. 4 bottom right panel).

\subsection{Similarity between two spike trains}

Following (Jolivet et al., 2008; Kobayashi et al., 2009) let us assume that spike trains $A$ and $B$ contain $N_{A}$ and $N_{B}$ spikes, and $M$ spikes occur at the same time in $A$ and $B$ with jitter $\Delta$. Then, the similarity between $A$ and $B$ is defined by the "coincidence factor" $\Gamma$ :

$$
\Gamma=\frac{100}{C_{\Gamma}} \frac{2(M-E)}{N_{A}+N_{B}}
$$

where $E=2 f \Delta N_{B}$ is the expected number of coincidences generated by a Poisson process with the same mean firing rate $f$ of spike train $B$. The jitter $\Delta$ is $3 m s$ here. $C_{\Gamma}$ is a normalization coefficient given by $(1-2 f \Delta)$ so that the coincidence factor is equal to $100(\Gamma=100)$ for two identical spike trains.

In this work, we calculated the coincidence factor between the original input spike train (spike train $A$ ) and spike trains reconstructed from neural output spike trains (spike train $\tilde{B}$ ). The delay produced by neuronal dynamics and synaptic transmission must be taken into account in order to calculate precisely the coincidence factor. We calculated the cross-correlogram from spike train $A$ to $\tilde{B}$, and defined the delay by the time at the peak of the correlogram. Then we shifted the spike train $\tilde{B}$ backward by this delay, yielding the spike train $B$, which was used to calculate the coincidence factor with the spike train $A$.

\section{Results}

Following the typical pattern of activity characteristic of excitatory feed-forward networks the neurons belonging to layers of higher order tended to be synchronized (Abeles, 1982a; Tetzlaff et al., 2002, 2003). This was true for networks formed by either type of neuron models (Fig. 5). However, it is interesting to notice that synfire activity was not necessarily associated to the external input spike train. In the examples shown in Fig. 5b with a background noise of 500 spikes $/ s$ many spurious synchronous events appeared in the 10th layer (L10) in the network composed of RS-EIF neurons. A spurious synfire event appeared in L10 near time $40240 \mathrm{~ms}$ for RS-MAT and near time $40930 \mathrm{~ms}$ for RS-IZH. The firing rates and the correlation with the timing of the events of the external input are discussed in the next subsections.

\subsection{Firing rate of neurons}

Each cell receives 15 specific afferents (from the external input for Layer 1 or from cells belonging to the previous layer for Layer 2 and beyond) and a background activity. In the case of a simulation with Chen's system with copy probability $\varepsilon=0.3$ and a background intensity of 500 spikes $/ \mathrm{s}$, a cell in Layer 1 received 575 spikes $/ s$ (equal to $5 \times 15+500$ spikes $/$ s) and generated an output spike train with an average rate of 5.4 spikes $/ s$ for RS-MAT neuronal model. In the case of the RS-MAT neuron in Layer 4 received 593 spikes $/ s$ (equal to $6.2 \times 15+500$ spikes $/ \mathrm{s}$ ), and fired at an average rate of 6.5 spikes $/ \mathrm{s}$.

We analyzed the mean firing rate of cells averaged in each layer as a function of the order of the layers. In case of Chen's system as source of dynamical system used to generate the original input spike train we showed that higher firing rates were observed with larger values of copy probability $\varepsilon$ (Fig. 6, left column). At a specific layer we observed that larger background activity induced higher firing rates for all tested neuronal models. Notice that for the current RS-EIF model even with copy probability $\varepsilon=1.0$ (Fig. 6a, top left) a background intensity of 400 spikes $/ s$ was necessary to obtain a stable activity along the downstream layers. With this RS-EIF model the activity could not propagate after Layer 6 with a background intensity of 300 spikes $/ \mathrm{s}$ and decreased by more than half at Layer 10 compared to Layer 1 with a background intensity of 350 spikes $/ \mathrm{s}$. On the opposite, a background intensity of 500 spikes $/ \mathrm{s}$ increased the firing rate along the downstream layers by more than $200 \%$ at Layer 10 (notice that the vertical scale for RS-EIF extends twice more than for RS-IZH and RS-MAT).

For RS-IZH neurons with Chen's system input (Fig. 6a, top left) the mean firing rate in each layer was almost constant or slightly increased throughout the layers. A similar trend was observed for RS-MAT neurons except with a background intensity of 500 spikes $/ \mathrm{s}$ characterized by a linear increase in firing rate, although much more moderate than for RS-IEF, along the downstream layers. With a copy probability $\varepsilon=0.3$ (Fig 6b, left column) the sensitivity to background intensity was bigger, in comparison to $\varepsilon=1.0$ (Fig 6a, left column), for all 

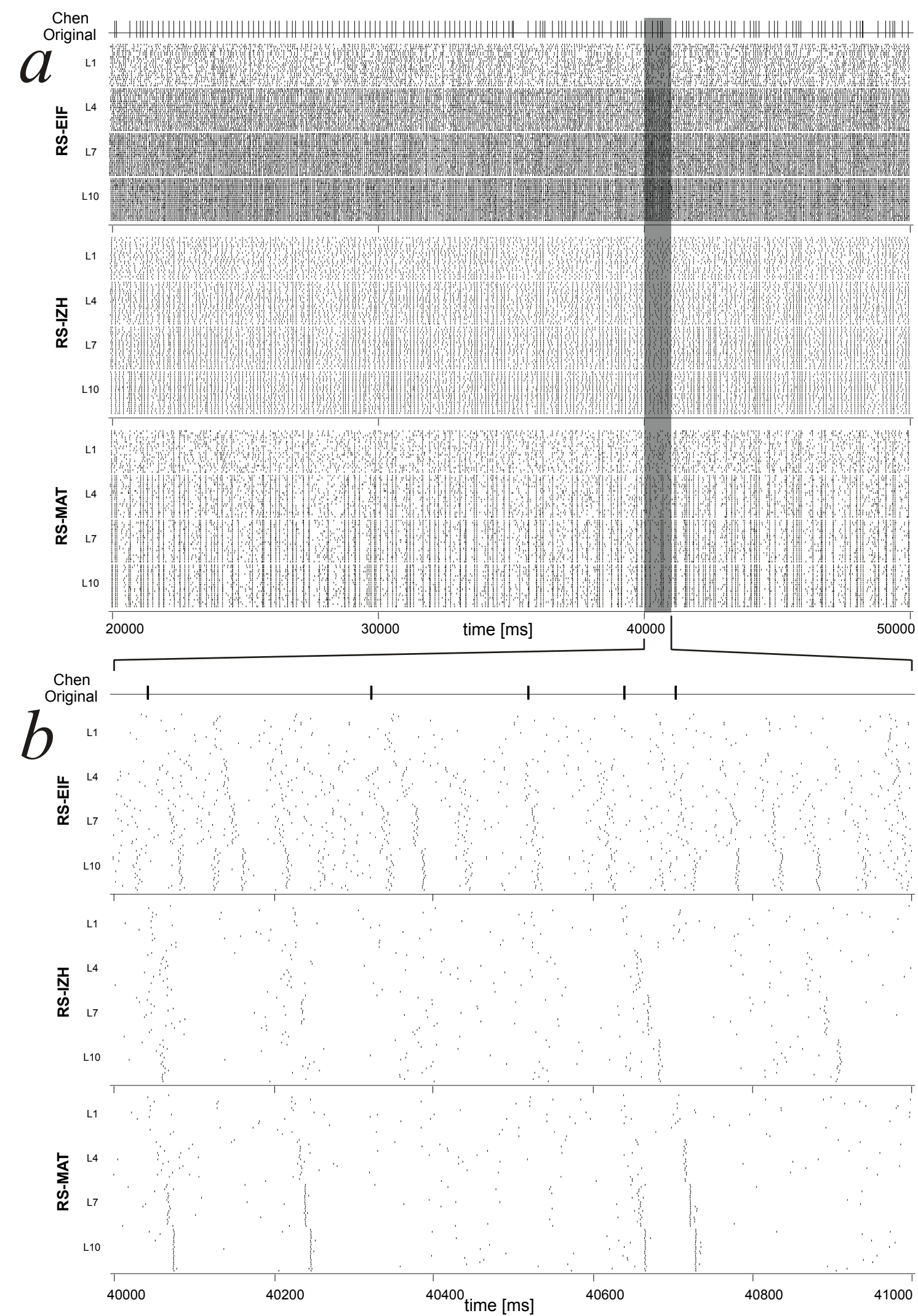

Figure 5: Raster plots of networks formed by either RS-EIF, RS-IZH, or RS-MAT neurons. The external input is generated by Chen's attractor with $\varepsilon=0.3$ and background intensity of 500 spikes/s. Each line of the raster represents the activity of a neuron and each dot represents the occurrence of a spike. Each raster represents the activity of 100 neurons: 20 cells of Layers 1,3,4,7 and 10 (L1,L4,L7,L10). Panel (a) shows the raster of the activity in the interval between time 20000 and $50000 \mathrm{~ms}$ of the simulation. The gray area represents the interval 40000-41000 that is represented at a larger time resolution in panel $(\mathrm{b})$. 

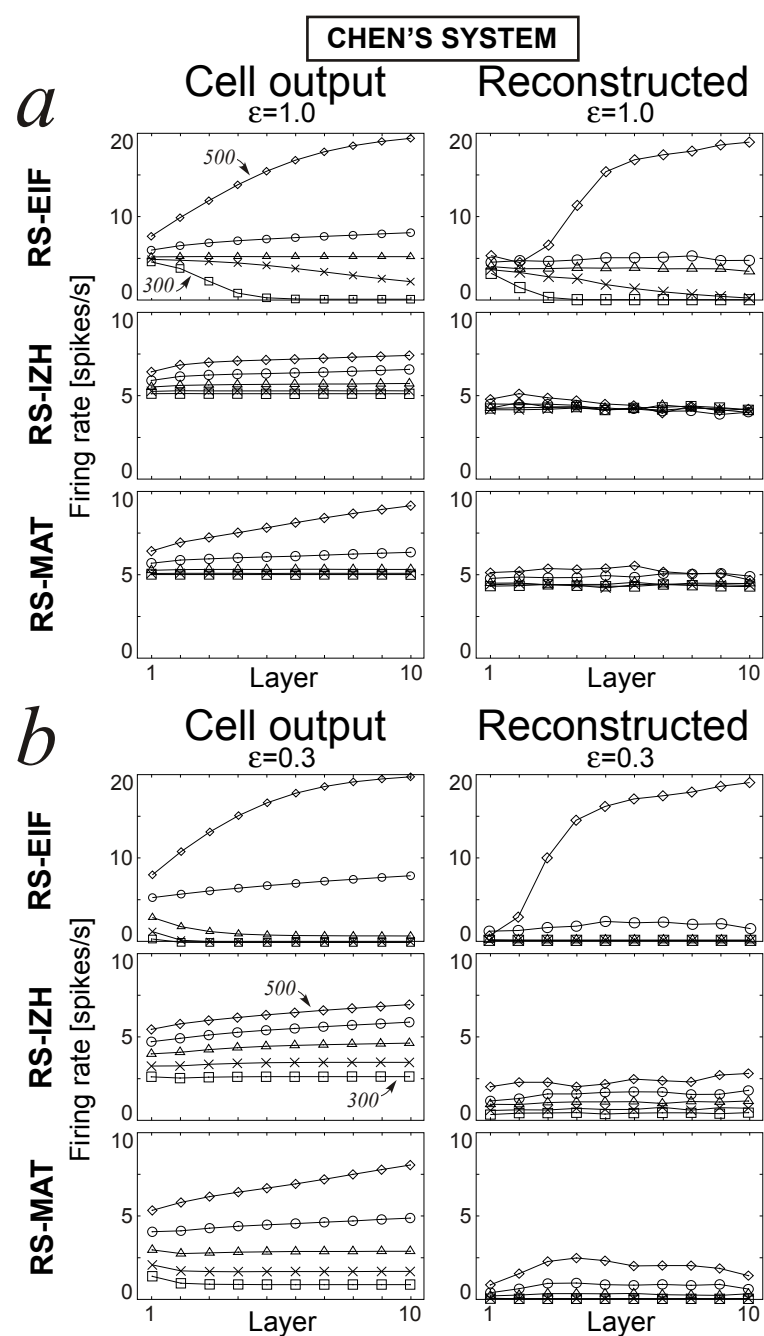

Figure 6: Mean firing rates of cells averaged in each layer for Chen's system as source dynamical systems to generate the original input spike trains with $(a)$ $\varepsilon=1.0$ and (b) $\varepsilon=0.3$ for of RS-EIF, RS-IZH, and RS-MAT models. The left column illustrates the firing rate calculated on the plain neuronal output spike train as a function of the order of the layer and of the background noise in the range $300-500$ spikes $/ \mathrm{s}$. Symbols square, cross, triangle, circle and diamond correspond to intensity 300,350, 400, 450 and 500 spikes/s, respectively.

models as indicated by a broader range of firing rates at Layer 10 between the minimum with 300 and the maximum with 500 spikes/s. However, for RS-EIF the sensitivity was so high that only a background intensity between 400 and 450 spikes $/ \mathrm{s}$ range could produce an almost constant sustainable mean firing rate throughout the layers. Notice that given a certain neuronal model and a copy probability $\varepsilon$ there was little difference in the profiles of the mean firing rates calculated on the total cell output between Chen's system and the other two dynamical systems.

The analysis of the firing rate of the reconstructed spike trains for Chen's system (Fig. 6, right column) and Ikeda map (Fig. 7) showed that the effect of background intensity on the number of spikes detected by PGA. was much smaller than for the total output spike train. In the case of Zaslavskii map (data not

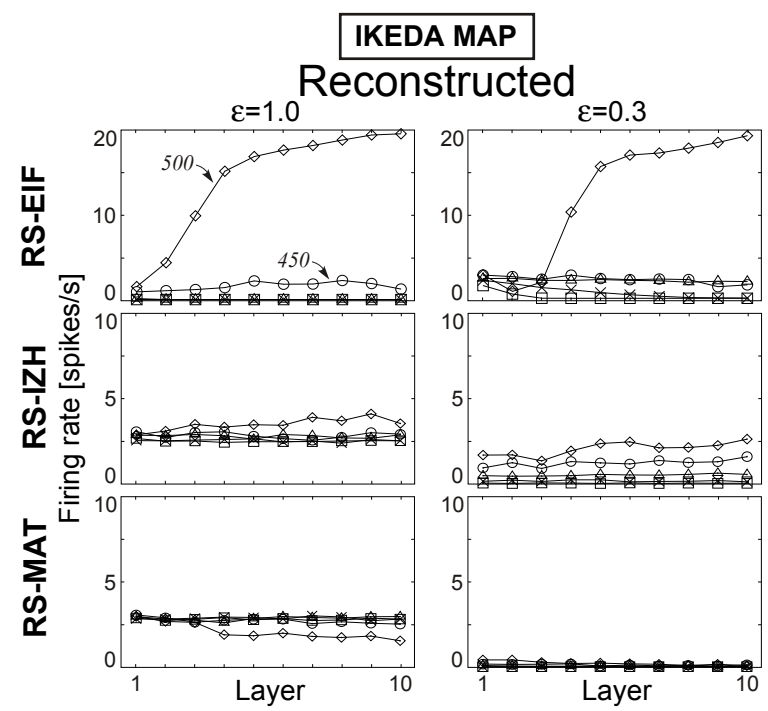

Figure 7: Mean firing rates of reconstructed spike trains in each layer for Ikeda map with $\varepsilon=1.0$ and $\varepsilon=0.3$ for of RS-EIF, RS-IZH, and RS-MAT models, as a function of the order of the layer and of the background noise in the range 300-500 spikes/s. Same symbols used in Figure 6.

shown), all profiles of the firing rate of the reconstructed spike train were similar to those of Chen's system. Notice that for reconstructed spike trains and a copy probability $\varepsilon=1.0$ the mean firing rate tended to be almost constant for any background activities for RS-IZH and RS-MAT models. According to the results obtained with the total output spike trains RS-EIF neurons needed a background intensity within a narrow range close to 450 spikes $/ \mathrm{s}$ to show a sustained and stable activity (Fig. 6a, top right panel; Fig. 7, top left panel). Below that intensity the activity was too low to grant the propagation of spatiotemporal patterns of spikes and at 500 spikes $/ \mathrm{s}$ the activity increased along the downstream layers and produced large amount of spurious patterns. With a copy probability $\varepsilon=0.3$ the mean firing rates were lower and the trend was similar to copy probability $\varepsilon=1.0$ except for RS-MAT neurons. For this neuronal model Chen's system and Zaslavskii inputs produced detectable firing patterns for background intensities above 400 spikes $/ \mathrm{s}$ (Fig. 6b, bottom right panel) but with Ikeda input almost no patterns could be detected (Fig. 7, bottom right panel).

\subsection{Cross-correlation analysis}

Let us consider a test condition with stable and sustained activity throughout the layers and yet with a large proportion of Poissonian spikes, such as Chen's system with copy probability $\varepsilon=0.3$ and background intensity of 450 spikes $/ s$ for RS-EIF and 500 spikes/s for RS-IZH and RS-MAT models. For all models we computed the cross-correlograms (CC) of the output spike train of a cell with all its input spikes (Fig. 8a,c,e) and the $\mathrm{CC}$ of the output spike train of a cell with the original external input spike train (Fig. 8b,d,f).

The correlation between the input and the output for a cell of Layer 1 showed that the latency of the CC peak was equal to $2 m s, 6 m s$, and $1 m s$ for RS-EIF, RS-IZH and RS-MAT models, 

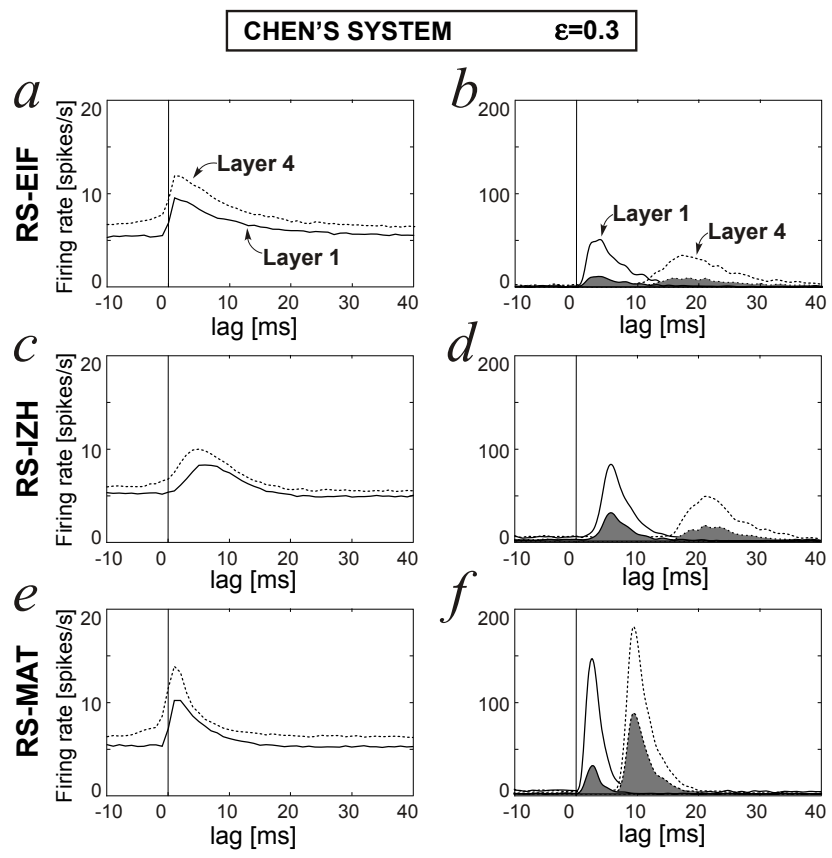

Figure 8: Propagation of activity in the network activated by an external input derived from Chen's system with $\varepsilon=0.3$ and background activity of 450 spikes $/$ s for RS-EIF and of 500 spikes $/ s$ for RS-IZH and RS-MAT. Crosscorrelation between all input spikes arriving at a cell and its output spike train for RS-EIF (panel $a$ ), RS-IZH (c), RS-MAT (e) models for one Layer 1 cell (solid line) and for Layer 4 cell (dashed line). Panels $b, d, f$ : Cross-correlation between the original external input spike train and the cell's output spike train (white area) for RS-EIF $(b)$, RS-IZH $(d)$, RS-MAT $(f)$ models for a Layer 1 cell (solid line) and for Layer 4 cell (dashed line). The shaded area refers to the cross-correlations between the original external input spike train and the cell's reconstructed spike train of the same Layer 1 and Layer 4 cells. Notice that the cross-correlograms are scaled in firing frequency units with a bin of $1 \mathrm{~ms}$ according to Abeles (1982b).

respectively (Fig. 8a,c,e). These latencies remained unchanged throughout the downstream layers and the $\mathrm{CC}$ between all input spikes and the output spike train of a Layer 4 cell showed latencies of the CC peaks equal to $2 \mathrm{~ms}, 5 \mathrm{~ms}$, and $1 \mathrm{~ms}$ for RSEIF, RS-IZH, and RS-MAT models, respectively (Fig. 8a,c,e). Notice that RS-EIF and RS-MAT models were characterized by very short and comparable latencies of the CC peak and similar asymmetry of the curves.

The cross-correlograms (CC) of the output spike train of Layer 1 and Layer 4 cells with the original external input spike train clearly shows asymmetric peaks for all neuronal models (Fig. 8b,d,f). The latencies of the CC peaks for the RS-EIF cells in Layer 1 and Layer 4 were equal to $4 \mathrm{~ms}$ and $17 \mathrm{~ms}$, respectively. For RS-IZH cells, the latencies were $6 \mathrm{~ms}$ and $21 \mathrm{~ms}$ for Layer 1 and Layer 4, respectively. For RS-MAT cells, the latencies were $3 \mathrm{~ms}$ and $9 \mathrm{~ms}$ for Layer 1 and Layer 4, respectively. The longer latencies of the $\mathrm{CC}$ peak for Layer 4 cells are due to the delay in information transmission corresponding to an increasing accumulation of the delays of synaptic transmission and integration of input synaptic currents along the layers of the network. It is interesting to notice that for RS-EIF and RS-IZH models the peaks of the CCs for Layer 4 cells with the original external input spike train were smaller than the CCs for Layer 1 cells (Fig. 8b,d). Conversely, RS-MAT showed higher peaks for Layer 4 than Layer 1 CCs (Fig. 8f). In addition, RSMAT showed the shortest latency despite a similar jitter to RSEIF in the input-output transfer latency (Fig. 8a,e). The CCs of the original external input with the cell's reconstructed output spike train (Fig. 8b,d,f, dashed areas) showed curves similar to the CCs with cell output, although with lower peaks. In particular, with RS-EIF model the peaks with the cell's reconstructed output spike train (Fig. 8b, dashed areas) were extremely low.

A signal-to-noise ratio ( $\mathrm{SNR}$ ) was calculated as $S N R=$ $\sqrt{\text { peak height/baseline }}$ where the baseline is defined as the average discharge rate measured on the $\mathrm{CC}$ between -20 and $0 \mathrm{~ms}$ time lag. Table 1 shows the SNRs for Chen's system with $\varepsilon=$ 0.3 and background activities equal to 450 and 500 spikes $/ \mathrm{s}$ of CCs with cell output and of CCs with the reconstructed output spike trains. Notice that for RS-EIF neurons a background of 500 spikes $/$ s produced many spurious firing patterns and does not represent a meaningful value for comparison. The SNRs for this model wee equal to the smallest values we have observed and tended to decrease by more than $25 \%$ from Layer 1 to Layer 4. For the RS-MAT neurons the SNR measured on the CCs calculated between the original input spike train and the reconstructed spike train tended to be larger than their corresponding values measured on the CCs with the total output spike train. Moreover, for the RS-MAT neurons, SNRs were larger at Layer 4 than at Layer 1 (see also Fig. 8f).

The improvement of the SNR in the reconstructed spike train over the cell output was assessed by SNR change $(\%)=$ $\left[\left(S N R_{\text {rec }} / S N R_{\text {cell }}\right)-1\right] \times 100$. In the absence of Poissonian inputs (i.e., $\varepsilon=1.0$ ) and background intensity equal to 400 spikes $/ \mathrm{s}$ no differences were observed among the three models (Fig. 9a). Notice that with the same background intensity and copy probability $\varepsilon=0.3$ the RS-EIF model was unable to generate sustainable activity throughout all layers and its corresponding curve was be displayed (Fig. 9b). SNR change tended to stay at low level and rather constant for RS-EIF and RS-IZH neurons (Fig. 9a). On the opposite, with an increase of the background intensity the SNR change tended to increase going downstream along the layers of the RS-MAT neurons net-

Table 1: Signal-to-noise ratio for Chen's system with $\varepsilon=0.3$ for all models and two background intensities calculated between the original input spike train and the cell output and between the original input spike train and the reconstructed output spike train.

\begin{tabular}{rrrrrr}
\hline \hline \multirow{2}{*}{ Model } & \multicolumn{2}{c}{ Background 450 spikes $/ s$} & & \multicolumn{2}{c}{ Background 500 spikes $/ s$} \\
\cline { 2 - 3 } & Layer 1 & Layer 4 & & Layer 1 & Layer 4 \\
\hline Cell output & & & & & \\
RS-EIF & 3.8 & 2.9 & & 3.2 & 1.8 \\
RS-IZH & 5.0 & 4.2 & & 4.4 & 3.2 \\
RS-MAT & 7.7 & 9.6 & & 6.5 & 7.0 \\
Reconstructed & & & & \\
RS-EIF & 3.9 & 2.9 & & 3.3 & 1.8 \\
RS-IZH & 5.1 & 4.9 & & 4.5 & 3.5 \\
RS-MAT & 9.8 & 13.7 & & 7.9 & 9.2 \\
\hline
\end{tabular}



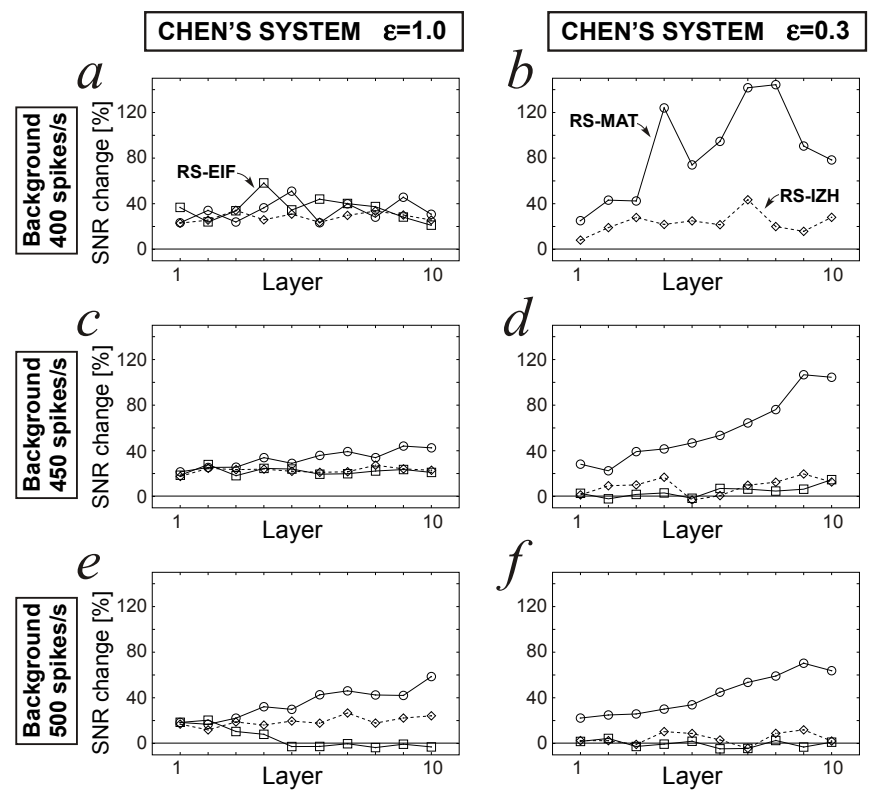

Figure 9: Changes in SNR (signal-to-noise ratio) from the cross-correlogram of total outputs to the cross-correlogram of reconstructed spike train at each layer for RS-EIF (solid curve, squares), RS-IZH (dashed curve, diamond symbols), and RS-MAT (solid curve, circles) models. Panels $(a),(c),(e)$ and $(b),(d),(f)$ correspond to results obtained with an input produced by Chen's system and copy probability $\varepsilon=1.0$ and $\varepsilon=0.3$, respectively. In panels $(a),(b)$ the networks received background intensities of 400 spikes/s $(a, b)$, of 450 spikes $/ \mathrm{s}$ $(c, d)$ and of 500 spikes $/ s(e, f)$.

work (Fig. 9). If one considers all simulations, regardless of the three dynamical systems studied here, regardless of the intensities of the background activity and regardless of the network layer we calculated a grand average of the SNR change and we observed that reconstructed spike trains exhibited an improvement in the SNR over the total output spike train by $21 \%$ for RS-EIF, by $25 \%$ for RS-IZH and by $30 \%$ for RS-MAT neurons.

\subsection{Coincidence analysis}

The coincidence factor is a measure of the preservation of the temporal structure fed into Layer 1 from outside the network. In case of external inputs generated with $\varepsilon=1.0$ no noise was introduced in the original input spike trains. For external input generated by Chen's system (as well as for Zaslavskii map, data not shown) the coincidence factor remained above a value of 80 from Layer 1 throughout all layers of the network for both RS-IZH and RS-MAT models if background intensities up to 400 spikes/s (Fig. 10a, left panels). On the opposite, with a background intensity of 500 spikes/s the value of the coincidence factor dropped to near 25 at Layer 10 for the RSIZH network but remained close to 75 for the RS-MAT network (Fig. 10a, left panels). For RS-EIF the coincidence factor stayed at a level above 50 only for background intensities $n$ the range 400-450 spikes / s, otherwise this factor decreased rapidly along the downstream layers.

In case of a large amount of noise present in the external input, e.g. with a copy probability $\varepsilon=0.3$, the coincidence factor
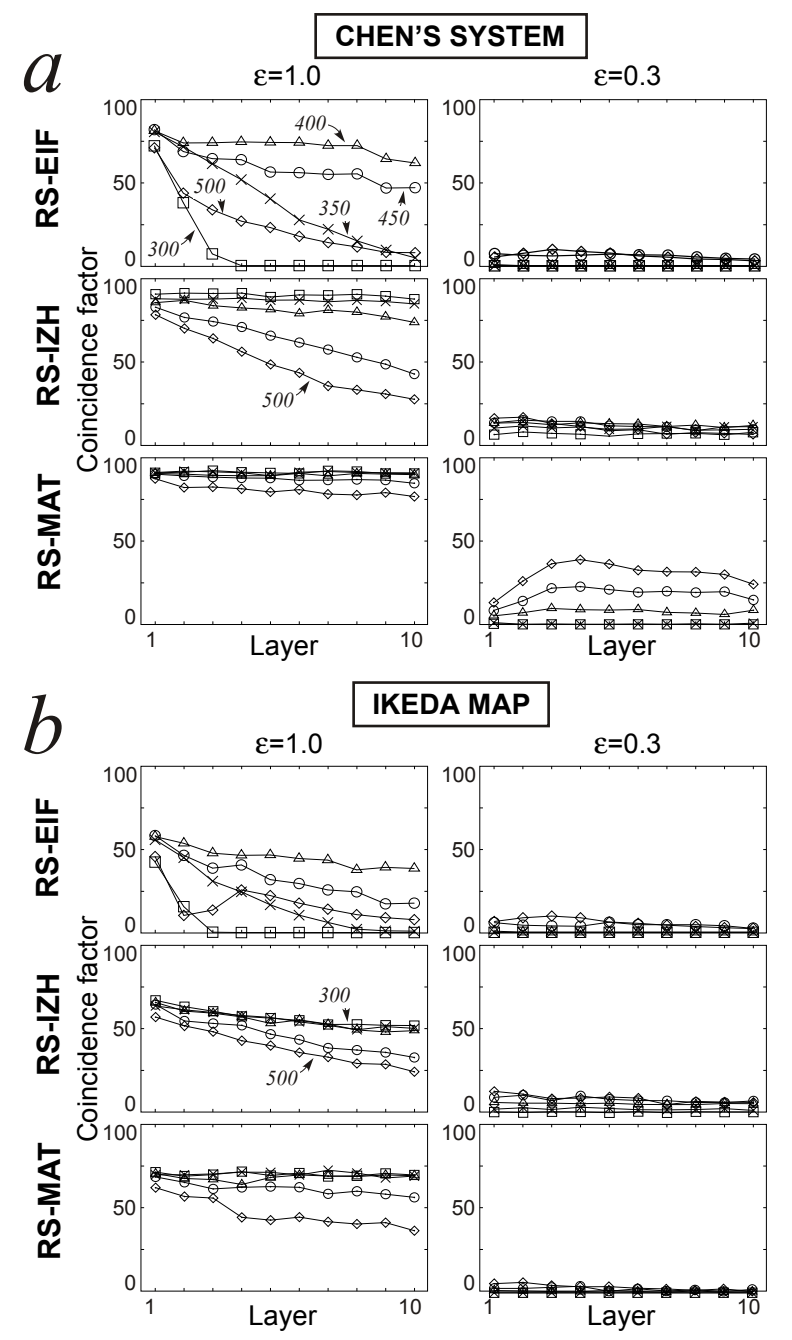

Figure 10: Coincidence factor calculated for a neuron at each layer as a function of the layer order for all values of background intensities. Symbols square, cross, triangle, circle and diamond correspond to background intensities of 300 , $350,400,450$ and 500 spikes $/$ s, respectively. The external input was generated by (a) Chen's system and (b) Ikeda map with $\varepsilon=1.0$ (left panels) and $\varepsilon=0.3$ (right panels).

was below 20 already at the level of Layer 1 for any kind of external input (Fig. 10a,b, right panels). However, in the case of Chen's system external input and with a network formed by RS-MAT neurons, the coincidence factor increased downstream and reached a maximum value at Layers 3-4 (Fig. 10a, bottom right panel). In this example for downstream layers beyond Layer 4 the value of the coincidence factor decreased again, but a value above 25 at Layer 10, still higher than at Layer 1 , was observed with a background intensity of 500 spikes/s. Such a non monotonic profile of the coincidence factor characterized by higher values near Layers 3-4 and higher values for certain higher levels of noise was observed also in the case of Zaslavskii map (data not shown).

In the case of Ikeda's external input the coincidence factor was below 75 at Layer 1 even with $\varepsilon=1.0$ for all models (Fig. 10b, left panels). Despite such low reading of the coin- 

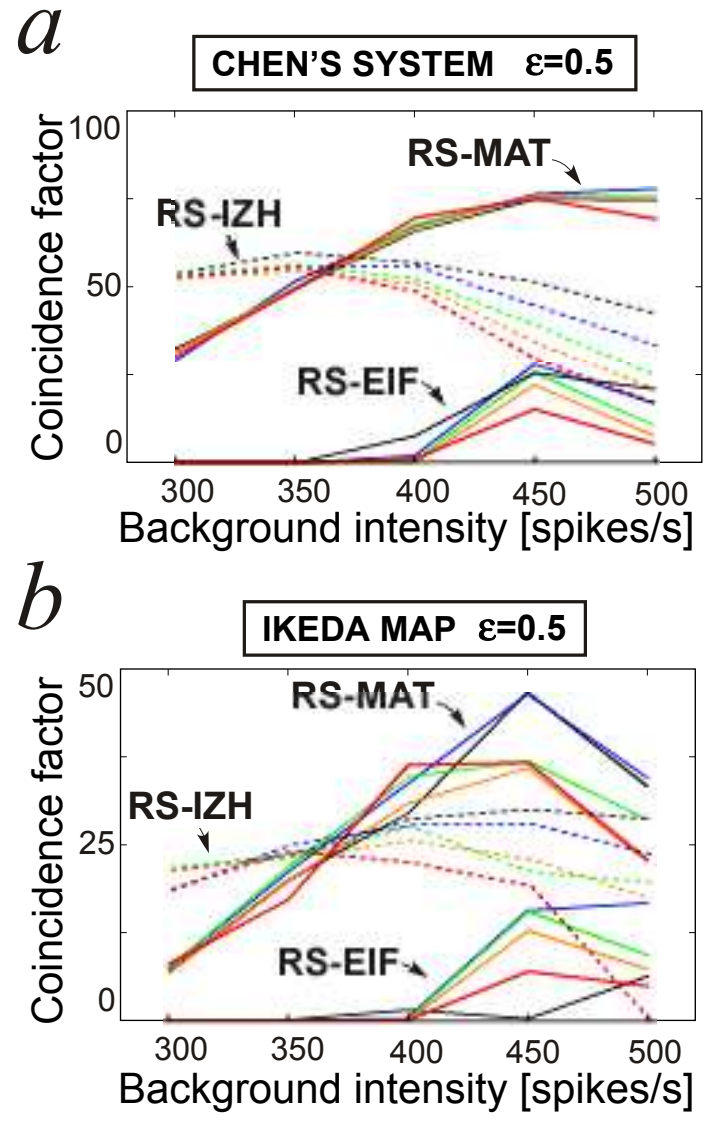

Figure 11: Coincidence factor calculated for a neuron of a layer as a function of background activity. The layers are color coded as follows: black-L2, blueL4, green-L6, orange-L8, and red-L10. The external input was generated by Chen's system $(a)$ and Ikeda map $(b)$ with $\varepsilon=0.5$. Non overlapping bundles of solid lines correspond to RS-EIF and RS-MAT models, as indicated by the legend in the graphic. Dashed lines correspond to RS-IZH. Notice that for RSMAT models higher coincidence factors were observed with larger background intensities.

cidence factor already at the entry of the network we observed that RS-MAT neurons tended to preserve its value in the downstream layers for background intensities up to 400 spikes $/ \mathrm{s}$. On the opposite, for RS-EIF and RS-IZH the coincidence factor tended to decrease along the networks with any background intensity. Interestingly, for $\varepsilon=0.3$ RS-EIF and RS-IZH neurons could sustain the coincidence factor (this value is low but higher than that of RS-MAT neurons) especially with background activity higher than 450 spikes $/ \mathrm{s}$ (Fig. 10b, right panels).

If a consistent amount of spikes unrelated to the nonlinear dynamics (e.g. $\varepsilon=0.5$ ) were fed to Layer 1 of RS-MAT network the value of the coincidence factor tended to increase with an increase of background activity (Fig. 11a,b). This property can be viewed as the ability of the network to produce a kind of de-noising process along the network layers. On the contrary, the coincidence factor for the RS-IZH neurons tended to decrease along the network layers with an increase of the background intensity for any value of $\varepsilon$, as exemplified with data obtained from Chen's system and $\varepsilon=0.5$ (Fig. 11a). In the
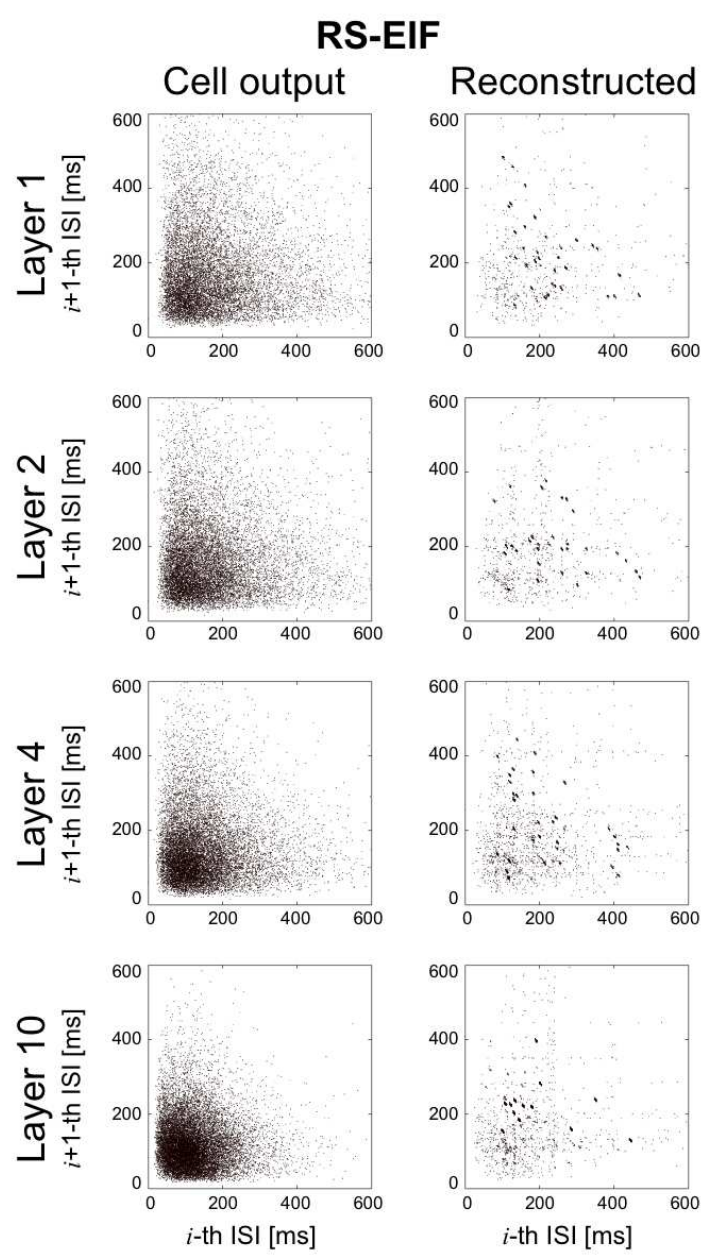

Figure 12: Examples of return maps of a single RS-EIF cell output and corresponding reconstructed spike trains. The original input is generated by a noisy Chen's system with $\varepsilon=0.3$ and background intensity of 500 spikes $/ \mathrm{s}$.

case of RS-EIF no sustainable activity could be observed below a background intensity of 400 spikes/s and the values of the coincidence factor for a given layer tended to be smaller than RS-IZH also with a background intensity of 500 spikes $/ \mathrm{s}$. It is worth to remark that a profile similar to a stochastic resonance could even be observed in the RS-MAT neural network with Ikeda map (Fig. 11b). In the absence of noisy inputs (e.g., for large values of copy probability $\varepsilon$ ) the coincidence factor is always better preserved along the network formed by RS-MAT neurons, irrespective of the origin of the temporal information fed to Layer 1 (Fig. 10).

The efficacy of the transmission of the temporal information through downstream layers can be observed in the return maps of neural outputs and reconstructed spike trains. For RS-EIF, RS-IZH, and RS-MAT networks Figures 12, 13 and 14, respectively, show examples of return maps in Layers 1, 2, 4, 10 in a case of a noisy external input $(\varepsilon=0.3)$ generated by Chen's system in presence of high background activity (500 spikes $/ \mathrm{s}$ ). The return maps of the RS-EIF and RS-IZH neuronal outputs show no recognizable shape of the attractor seen in the origi- 

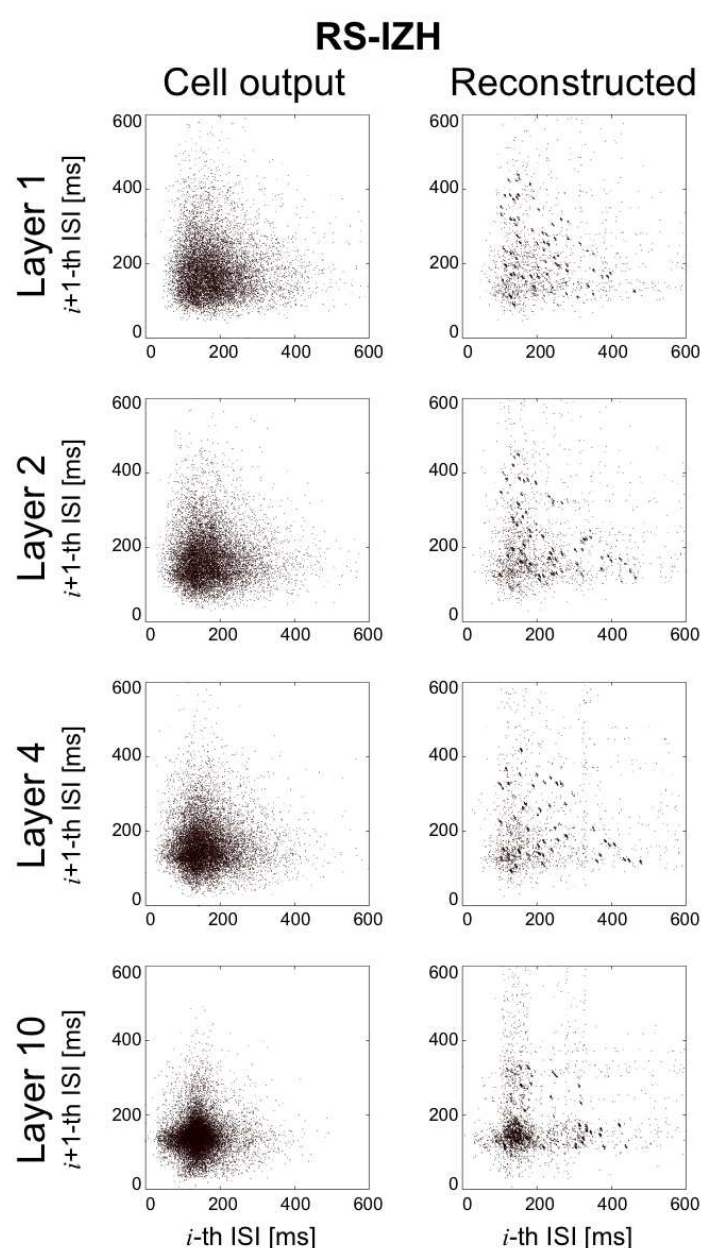

Figure 13: Examples of return maps of a single RS-IZH cell output and corresponding reconstructed spike trains. The original input is generated by a noisy Chen's system with $\varepsilon=0.3$ and background intensity of 500 spikes $/ \mathrm{s}$.

nal input spike train (Fig. 12 and Fig. 13, left panels). Notice that sizable amount of preferred firing sequences unrelated to the external input dynamics were detected by PGA in RS-IZH network, as shown by the return map of the reconstructed spike train of Layer 10 neurons (Fig. 13 bottom right panel), but those events were associated to the intrinsic dynamics of the neuronal model. On the contrary, for RS-MAT neurons the shape of the original attractor become clearer going downstream along the network layer in the analysis of cell output (Fig. 14 left panels). Return maps of the reconstructed RS-MAT spike trains emphasize even more the spikes associated to the external input dynamics, especially for Layer 4 characterized by the largest coincidence factor in this set of results.

\section{Discussion}

We observed the ability of partially convergent/divergent feed-forward neural networks to integrate and transmit temporal information embedded in a set of input spike trains in presence of stochastic background noise. The feature of this network structure in propagating synchronous firing (Abeles,
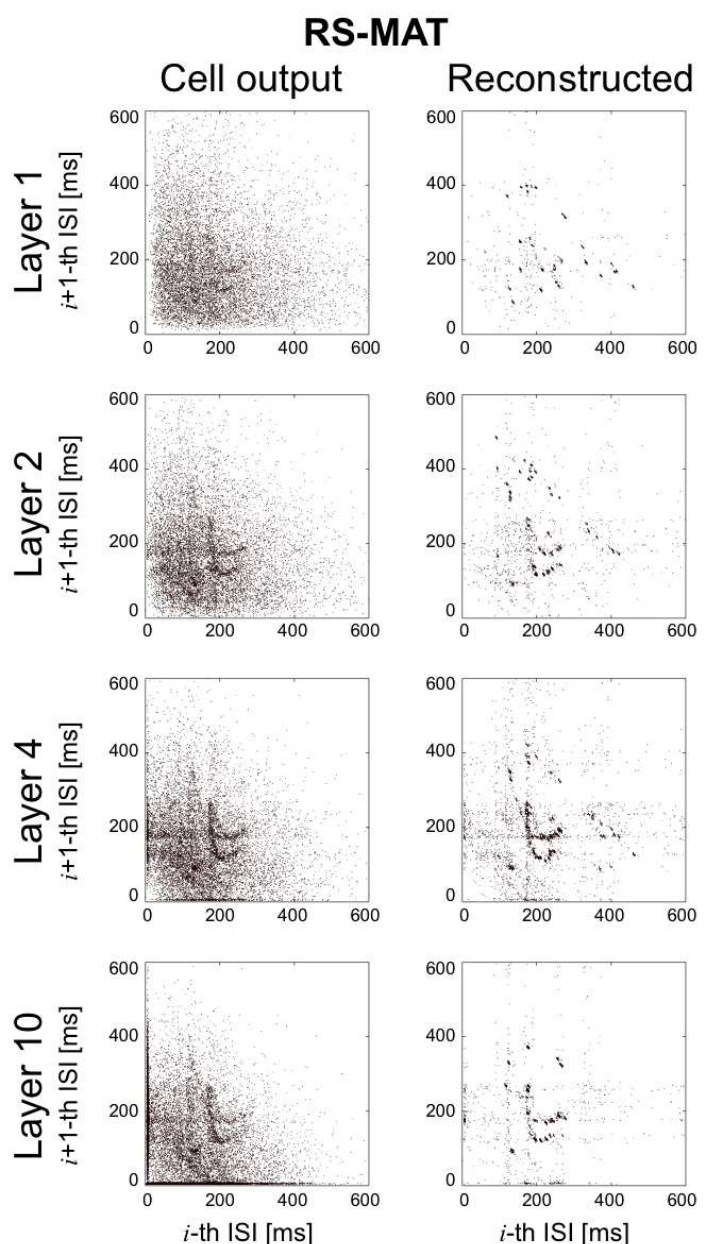

Figure 14: Examples of return maps of a single RS-MAT cell output and corresponding reconstructed spike trains. The original input is generated by a noisy Chen's system with $\varepsilon=0.3$ and background intensity of 500 spikes $/ \mathrm{s}$.

1982a; Diesmann et al., 1999) can be used to preserve and transmit much of a distributed temporal information, even for noisy external input fed at the entry of the network, at least up to 10th order of the layered structure. We show that recurring spike patterns detected downstream along the network layers result not only from the architecture of the network but a sizable amount of recurring patterns result from the temporal structure of the original input as a function of the dynamics underlying the temporal structure of the input, of the selected neuron model and of background noise intensity.

Large values of copy probability $\varepsilon$ mean that a neuron of the input layer (Layer 1) receives more spikes derived from the original input spike train. In this case, input spikes to Layer 1 belonging to the original input spike train have a heavier weight than Poisson distributed afferent spikes. These spikes can be more efficient in driving the postsynaptic neurons in the first layer in presence of a proper level of membrane excitability maintained by an adequate background activity, thus resulting in higher response rates (Fig. 6). The procedure of copying spikes from a "mother process" with some probability was pre- 
viously used by Kuhn et al. (2003). The input spikes used in the current study is rather similar to their MIP correlated input. Kuhn et al. (2003) showed that when the input population size was rather small, the output neuronal firing rate increased almost linearly with an increase of the correlation coefficient among the input spike trains. For large sizes of the input population, the output rate showed a unimodal response as a function of the correlation coefficient. Notice that in this study we used fifteen out of twenty inputs converging to a single neuron of a layer, thus corresponding to the smaller input population considered by Kuhn et al. (2003).

The pattern grouping algorithm PGA (Villa and Tetko, 1999; Tetko and Villa, 2001b,c,a; Abeles and Gat, 2001) could detect almost a constant amount of spikes with $\varepsilon=1.0$ throughout all layers of the network for both RS-IZH and and RS-MAT neural models despite an increase of the average discharge rate along the downstream layers and for larger background activities. The RS-EIF was much more sensitive to background intensities than RS-IZH and RS-MAT and more than 400 spikes $/ \mathrm{s}$ were necessary for a sustainable propagation of synfire activity to the downstream layers and, in particular, for the detection of recurrent firing patterns with an input derived from Ikeda map. Notice that the parameters that were set in PGA to search for the preferred firing sequences appear to be less adequate to detect the dynamics of the Ikeda map than for Zaslavskii and Chen's system (Asai and Villa, 2008).

In the case of RS-EIF model the coincidence factor tended to be lower than for the other models, for comparable background intensities and copy probability. Notice that networks formed by RS-EIF neurons produced a large amount of synfire activity at downstream layers (e.g., Fig. 5) that was mainly unrelated to the original input generated by dynamical systems (e.g., Fig. 12). For RS-IZH model, the coincidence factor declined along the downstream layers despite a sustained firing activity and amount of patterns detected by PGA (e.g., Fig. 13). Our results suggest that a network composed by RS-MAT neurons (Kobayashi et al., 2009) can integrate and retain the temporal information embedded in an external input better than a network composed by RS-IZH neurons (Izhikevich, 2004), which are likely to generate preferred firing patterns that are not associated to the original external input, as suggested elsewhere (Asai et al., 2008).

The RS-EIF model is a good candidate to capture spike generation dynamics described by Hodgkin-Huxley-type conductance-based models (Fourcaud-Trocmé et al., 2003). We used the parameters as originally indicated by the authors but other sets of parameters have been suggested (Badel et al., 2008; Ostojic et al., 2009), emphasizing, in particular, a sharper initiation of the action potential. However, notice that networks of RS-EIF model exhibited a behavior similar to RS-IZH for some features and similar to RS-MAT for other features, as shown by the analysis of the cross-correlations (Fig. 8). In the RS-MAT model a spike is generated instantly once the threshold is crossed, thus responding quickly to a fast input transient such as a volley of synchronous spikes. Volleys of near synchronous spikes may propagate through the network even if synchrony in the first layer is not perfect (e.g., see Fig. 5) as described by Diesmann et al. (1999). Conversely, the RS-IZH model constitutes the normal form of a simplified HodgkinHuxley-type model aimed at studying the bifurcations and the origin of different response properties of different cell types. The quadratic nonlinearity of the membrane potential characteristic of RS-IZH makes its spike-onset much slower than other realistic models (Fourcaud-Trocmé et al., 2003; Naundorf et al., 2006), thus affecting the response speed and precision of a neuronal population formed by this type of neurons (Naundorf et al., 2005). On the basis of the analysis of the coincidence factor (Fig. 11) a kind of hierarchy tended to appear with respect to the level of background intensity. However, despite the fact that all models reproduced the dynamics of regular spiking neurons in a fair way, we cannot discard that a fine tuning of their parameters could produce results for RS-EIF and RS-IZH closer to RS-MAT.

With small values of $\varepsilon$, spikes with stochastic timing are dominant and the short intervals embedded in the temporal information tend to be lost. However, the structure itself of convergent/divergent feed-forward network is shaped to decrease synaptic jitter and preserve very precisely the firing times of the afferents (Abeles, 1982a). In a theoretical study it was reported that background activity allows slightly subthreshold activity to propagate reliably but not with high temporal precision (Stimberg et al., 2007), but experimental observations suggested that subthreshold temporally organized activity could be preserved to a large extent (Takahashi et al., 2010). The way by which noise is introduced in the network is also important. On the one side the introduction of jittered synaptic delays instead of random noise inputs is likely to produce similar results (Ikeda, 2003). On the other side it has been reported that feed-forward networks with unreliable synapses behave differently from the same networks randomly connected with reliable synapses in order to preserve the same connection density (Guo and Li, 2011).

It is interesting to notice that for values of copy probability $\varepsilon$ in the range $0.3-0.5$ we observed (in RS-MAT neural networks in the cases of external inputs generated by Chen's system and Zaslavskii map) that higher background activity facilitated the integration and transmission of the temporal information assessed by the coincidence factor (e.g., Fig. 10a). This phenomenon could be viewed as a kind of neuronal stochastic resonance (Bulsara et al., 1991; Longtin, 1993) in neural networks, when elevated excitability provided by the background activity can lead to the firing of spikes associated to a temporal attractor which is unable by itself to evoke post synaptic discharges. Figure $11 \mathrm{~b}$ shows more clearly the typical hallmark of the stochastic resonance which exhibits the peak of the coincidence factor at a certain intensity of the background activity, somehow in agreement with the observation of noise-enhanced propagation of activation waves in a synfire chain (Postma et al., 1996). Our previous experimental results (Villa et al., 1998a, 1999) showed that synchronized firing as well as complex spatiotemporal firing sequences were associated to behaviorally relevant activity, in agreement with the suggestion by Postma et al. (1996) that encoding mechanisms may produce patterns represented by the relative timing of action potentials. How- 
ever, they emphasized the transformation of an incoming spatiotemporal pattern into new spatiotemporal patterns as a consequence of the synaptic processes occurring throughout the network along the downstream layers. We support this hypothesis for some networks such as the basal ganglia-thalamo-cortical circuit, but the current study points out that (e.g., at corticocortical level) the propagation of synfire activity may transmit and preserve complex dynamics fed to the input layer of a partial diverging/converging neuronal network.

It is important to notice that different dynamical systems used so far gave rise to different performances. The relation between the properties of the dynamical systems such as the dimensions of the embedded space, of the attractor and the Lyapunov exponents with the network structure are very important elements to take into account. At this stage of our work we cannot speculate further on this observation because the optimal intensity of the background activity is likely to depend on the characteristics of the underlying dynamical attractor, the number of afferents converging to a neuron across the layer, and the copy probability $\varepsilon$. These are crucial questions that need to be addressed in further studies in order to develop a comprehensive theory that embrace dynamical systems and convergent/divergent feed-forward networks.

This study is the first, to our knowledge, that uses RSMAT class of models to study information processing in a feed-forward neuronal architecture. We suggest that multipletimescale adaptive threshold neurons are well suited for the integration and propagation of a distributed deterministic temporal information and preserve its dynamics through networks of cell assemblies in a robust way against background activities. A very recent evolution of the original RS-MAT model makes it even more interesting given its improved goodness of fit to experimental data (Yamauchi et al., 2011). Another important aspect to consider is the contribution of inhibitory cells in preferred firing sequences (Villa, 2000; Guo and Li, 2011) in balancing the activity of synfire chains (Aviel et al., 2003) and in controlling the propagation of synchronous volleys of activity (Shinozaki et al., 2007). Our further work is aimed at determining the limits and robustness of this performance by studying a broader set of networks with larger sizes of the layers, including inhomogeneous and diverging/converging networks with recurrent connections and with the introduction of synaptic plasticity.

\section{Acknowledgments}

The authors wish to acknowledge Professor S. Shinomoto, Dr. R. Kobayashi and the Reviewers for their comments, whose insight has contributed to expand the discussion and made the presentation clearer. This study was partially funded by the binational JSPS/INSERM grant SYRNAN and Japan-France Research Cooperative Program.

\section{References}

Abeles, M., 1982a. Local Cortical Circuits. Studies of brain function ; vol. 6 ; 102 p., Springer Verlag, Berlin ; New York.
Abeles, M., 1982b. Quantification, smoothing, and confidence limits for singleunits' histograms. J Neurosci Methods 5, 317-325.

Abeles, M., Gat, I., 2001. Detecting precise firing sequences in experimental data. J Neurosci Methods 107, 141-154.

Abeles, M., Hayon, G., Lehmann, D., 2004. Modeling compositionality by dynamic binding of synfire chains. J Comput Neurosci 17, 179-201.

Asai, Y., Guha, A., Villa, A.E.P., 2008. Deterministic neural dynamics transmitted through neural networks. Neural Netw 21, 799-809.

Asai, Y., Villa, A.E.P., 2008. Reconstruction of underlying nonlinear deterministic dynamics embedded in noisy spike train. J Biol Phys 34, 325-340.

Asai, Y., Villa, A.E.P., 2010. Transmission of distributed deterministic temporal information through a diverging/converging three-layers neural network. Lecture Notes in Computer Sciences 6532, 145-154.

Asai, Y., Yokoi, T., Villa, A.E.P., 2006. Detection of a dynamical system attractor from spike train analysis. Lecture Notes in Computer Sciences 4131, 623-631.

Aviel, Y., Mehring, C., Abeles, M., Horn, D., 2003. On embedding synfire chains in a balanced network. Neural Comput 15, 1321-1340.

Badel, L., Lefort, S., Brette, R., Petersen, C.C., Gerstner, W., Richardson, M.J., 2008. Dynamic i-v curves are reliable predictors of naturalistic pyramidalneuron voltage traces. J Neurophysiol 99, 656-666.

Bulsara, A., Jacobs, E.W., Zhou, T., Moss, F., Kiss, L., 1991. Stochastic resonance in a single neuron model: theory and analog simulation. J Theor Biol 152, 531-55.

Celletti, A., Froeschlé, C., Tetko, I.V., Villa, A.E.P., 1999. Deterministic behaviour of short time series. Meccanica 34, 145-152.

Celletti, A., Villa, A.E.P., 1996a. Determination of chaotic attractors in the rat brain. J. Stat. Physics 84, 1379-1385.

Celletti, A., Villa, A.E.P., 1996b. Low dimensional chaotic attractors in the rat brain. Biol Cybern 74, 387-394.

Diesmann, M., Gewaltig, M.O., Aertsen, A., 1999. Stable propagation of synchronous spiking in cortical neural networks. Nature 402, 529-533.

Fourcaud-Trocmé, N., Hansel, D., van Vreeswijk, C., Brunel, N., 2003. How spike generation mechanisms determine the neuronal response to fluctuating inputs. J Neurosci 23, 11628-40.

Fourcaud-Trocmé, N., Hansel, D., van Vreeswijk, C., Brunel, N., 2003. How spike generation mechanisms determine the neuronal response to fluctuating inputs. J Neurosci 23, 11628-11640.

Guo, D., Li, C., 2011. Signal propagation in feedforward neuronal networks with unreliable synapses. J Comput Neurosci 30, 567-587.

Ikeda, K., 2003. A synfire chain in layered coincidence detectors with random synaptic delays. Neural Netw 16, 39-46.

Izhikevich, E.M., 2003. Simple model of spiking neurons. IEEE Transactions on Neural Networks 14, 1569- 1572.

Izhikevich, E.M., 2004. Which model to use for cortical spiking neurons? IEEE Trans Neural Netw 15, 1063-1070.

Jolivet, R., Kobayashi, R., Rauch, A., Naud, R., Shinomoto, S., Gerstner, W., 2008. A benchmark test for a quantitative assessment of simple neuron models. J Neurosci Methods 169, 417-24.

Kobayashi, R., Tsubo, Y., Shinomoto, S., 2009. Made-to-order spiking neuron model equipped with a multi-timescale adaptive threshold. Front Comput Neurosci 3, 9 .

Kuhn, A., Aertsen, A., Rotter, S., 2003. Higher-order statistics of input ensembles and the response of simple model neurons. Neural Comput 15, 67-101.

Kumar, A., Rotter, S., Aertsen, A., 2008. Conditions for propagating synchronous spiking and asynchronous firing rates in a cortical network model. J Neurosci 28, 5268-80.

Longtin, A., 1993. Stochastic resonance in neuron models. J. Stat. Physics 70, 309-327.

Naundorf, B., Geisel, T., Wolf, F., 2005. Action potential onset dynamics and the response speed of neuronal populations. J Comput Neurosci 18, 297 309.

Naundorf, B., Wolf, F., Volgushev, M., 2006. Unique features of action potential initiation in cortical neurons. Nature 440, 1060-1063.

Ostojic, S., Brunel, N., Hakim, V., 2009. How connectivity, background activity, and synaptic properties shape the cross-correlation between spike trains. J Neurosci 29, 10234-10253.

Postma, E.O., van den Herik, H.J., Hudson, P.T., 1996. Robust feedforward processing in synfire chains. Int J Neural Syst 7, 537-542.

Schrader, S., Diesmann, M., Morrison, A., 2011. A compositionality machine realized by a hierarchic architecture of synfire chains. Front Comput Neu- 
rosci 4, 154-154.

Segundo, J.P., 2003. Nonlinear dynamics of point process systems and data. International Journal of Bifurcation and Chaos 13, 2035-2116.

Segundo, J.P., Sugihara, G., Dixon, P., Stiber, M., Bersier, L.F., 1998. The spike trains of inhibited pacemaker neurons seen through the magnifying glass of nonlinear analyses. Neuroscience 87, 741-766.

Shinozaki, T., Câteau, H., Urakubo, H., Okada, M., 2007. Controlling synfire chain by inhibitory synaptic input. Journal of the Physical Society of Japan 76,044806 .

Stimberg, M., Hoch, T., Obermayer, K., 2007. The effect of background noise on the precision of pulse packet propagation in feed-forward networks. Neurocomputing 70, 1824-1828.

Takahashi, N., Sasaki, T., Matsumoto, W., Matsuki, N., Ikegaya, Y., 2010. Circuit topology for synchronizing neurons in spontaneously active networks. Proc Natl Acad Sci U S A 107, 10244-10249.

Tetko, I.V., Villa, A.E., 1997. A comparative study of pattern detection algorithm and dynamical system approach using simulated spike trains. Lecture Notes in Computer Science 1327, 37-42.

Tetko, I.V., Villa, A.E., 2001a. Pattern grouping algorithm and de-convolution filtering of non-stationary correlated poisson processes. Neurocomputing $38-40,1709-1714$.

Tetko, I.V., Villa, A.E.P., 2001b. A pattern grouping algorithm for analysis of spatiotemporal patterns in neuronal spike trains. 1. detection of repeated patterns. J. Neurosci. Meth. 105, 1-14.

Tetko, I.V., Villa, A.E.P., 2001c. A pattern grouping algorithm for analysis of spatiotemporal patterns in neuronal spike trains. 2. application to simultaneous single unit recordings. J. Neurosci. Meth. 105, 15-24.

Tetzlaff, T., Buschermöhle, M., Geisel, T., Diesmann, M., 2003. The spread of rate and correlation in stationary cortical networks. Neurocomputing 52-54, $949-954$

Tetzlaff, T., Geisel, T., Diesmann, M., 2002. The ground state of cortical feedforward networks. Neurocomputing 44-46, $673-678$.

Villa, A.E.P., 2000. Empirical evidence about temporal structure in multi-unit recordings, in: Miller, R. (Ed.), Time and the Brain. Harwood Academic Publishers. chapter 1, pp. 1-51.

Villa, A.E.P., Hyland, B., Tetko, I.V., Najem, A., 1998a. Dynamical cell assemblies in the rat auditory cortex in a reaction-time task. Biosystems 48 , 269-277.

Villa, A.E.P., Tetko, I.V., 1999. Spatiotemporal activity patterns detected from single cell measurements from behaving animals. Proceedings SPIE 3728, 20-34.

Villa, A.E.P., Tetko, I.V., Celletti, A., Riehle, A., 1998b. Chaotic dynamics in the primate motor cortex depend on motor preparation in a reaction-time task. Current Psychology of Cognition 17, 763-780.

Villa, A.E.P., Tetko, I.V., Hyland, B., Najem, A., 1999. Spatiotemporal activity patterns of rat cortical neurons predict responses in a conditioned task. Proceedings of the National Academy of Sciences of the USA 96, 1006-1011.

van Vreeswijk, C., Sompolinsky, H., 1996. Chaos in neuronal networks with balanced excitatory and inhibitory activity. Science 274, 1724-6.

Yamauchi, S., Kim, H., Shinomoto, S., 2011. Elemental spiking neuron model for reproducing diverse firing patterns and predicting precise firing times. Front Comput Neurosci 5, 42. 